\title{
Multihop Diversity in Wireless Relaying Channels
}

\author{
John Boyer, Student Member, IEEE, David D. Falconer, Fellow, IEEE, and Halim Yanikomeroglu, Member, IEEE
}

\begin{abstract}
This paper presents theoretical characterizations and analysis for the physical layer of multihop wireless communications channels. Four channel models are considered and developed: the decoded relaying multihop channel; the amplified relaying multihop channel; the decoded relaying multihop diversity channel; and the amplified relaying multihop diversity channel. Two classifications are discussed: decoded relaying versus amplified relaying, and multihop channels versus multihop diversity channels. The channel models are compared, through analysis and simulations, with the "singlehop" (direct transmission) reference channel on the basis of signal-to-noise ratio, probability of outage, probability of error, and optimal power allocation. Each of the four channel models is shown to outperform the singlehop reference channel under the condition that the set of intermediate relaying terminals is selected intelligently. Multihop diversity channels are shown to outperform multihop channels. Amplified relaying is shown to outperform decoded relaying despite noise propagation. This is attributed to the fact that amplified relaying does not suffer from the error propagation which limits the performance of decoded relaying channels to that of their weakest link.
\end{abstract}

Index Terms-Ad-hoc networks, cooperative diversity, diversity techniques, mesh networks, multihop channels, multihop diversity, wireless relaying.

\section{INTRODUCTION}

W IRELESS relaying networks allow mobile terminals to participate in the transmission of information when they are neither the initial source nor the final destination. This relaying can be applied to cellular, WLAN, ad-hoc, and hybrid networks in order to increase coverage, throughput, and capacity [10], [13], [14], [19], [24], [27]-[29]. Relaying systems realize a number of benefits over traditional systems in the areas of deployment, connectivity, adaptability, and capacity [13], [14]. Minimizing the need for fixed infrastructure results in networks that are easier, faster, and cheaper to deploy. The coverage of existing networks can be improved by extending the periphery and closing internal gaps [10]. Mesh connectivity, adaptive routing, and load balancing lead to networks that are inherently robust and adaptable to changing environments [24]. Shorter transmission distances and more efficient use of transmitted signal energy result in lower power levels throughout the system [19],

Paper approved by M.-S. Alouini, the Editor for Modulation and Diversity Systems of the IEEE Communications Society. Manuscript received May 7, 2003; revised January 16, 2004. This paper was presented in part at the IEEE Global Communications Conference, San Antonio, TX, November 25-29, 2001, in part at the 12th International Symposium on Personal, Indoor, and Mobile Radio Communications, San Diego, CA, September 30-October 3, 2001, and in part at the 7th Canadian Workshop on Information Theory, Vancouver, BC, Canada, June 4-6, 2001.

The authors are with the Broadband Communications and Wireless System (BCWS) Centre, Department of Systems and Computer Engineering, Carleton University, Ottawa, ON K1S 5B6, Canada (e-mail: jboyer@sce.carleton.ca; ddf@sce.carleton.ca; halim@sce.carleton.ca).

Digital Object Identifier 10.1109/TCOMM.2004.836447
[27], [28]. This implies increased system capacity, decreased interference levels, reduced terminal radiation, and longer battery life. The application of various spatial-diversity techniques enabled by the mesh connectivity of the mobile terminals has the potential to further compound these improvements.

Recently, cooperation between pairs of mobile terminals has been proposed in cellular and ad-hoc networks as a method of achieving the benefits of spatial diversity without requiring the use of physical antenna arrays [19], [20], [22], [27], [28]. These cooperation protocols involve the sharing of information on an intermobile channel, such that each user sends information using both of the terminals. Each transmission period is divided into two slots, where in the first slot, each terminal transmits its own information directly, and in the second slot, each terminal retransmits a portion of the partner's information. In general, these protocols are shown to improve the capacity and outage performance of the system.

The referenced cooperative protocols involve only a single intermediate relay terminal between the source and destination terminals. These protocols can be easily extended to the case of multiple relaying terminals in parallel [21], or multihop scenarios where each destination is instead a relay itself, and is paired with a different cooperating terminal with which it cooperates to transmit to the actual destination or next relay. However, there is no obvious extension to multihop cooperation scenarios where the signal from the source is directly received and used by many relays further along the transmission path, nor to asymmetric multihop cooperation scenarios where a relay further along the transmission path does not use the source to relay information to its destination. This paper introduces the concept of multihop diversity, where the benefits of spatial diversity are achieved from the concurrent reception of signals that have been transmitted by multiple previous terminals along a single primary route. This scheme exploits the broadcast nature of wireless networks where the communications channel is shared between multiple terminals. This form of spatial diversity is especially applicable to packet relaying [17], [18] and multihop ad-hoc networks [13], [14], since diversity gain can be achieved with minimal impact to existing serial relaying transmission protocols [4], [5], [15], [16] that already involve each terminal along the multihop path retransmitting the original signal. This diversity scheme contrasts with the scheme proposed in [2] and [6], where spatial diversity is achieved by relaying the signal along multiple routes in parallel. The performance of serial relaying without diversity is also studied in [11] and [31].

This paper considers four channel models for the case where mobile terminals act as intermediate relays in wireless communications systems. The decoded relaying multihop channel corresponds to the case where each intermediate terminal digitally decodes and re-encodes the received signal from the immedi- 


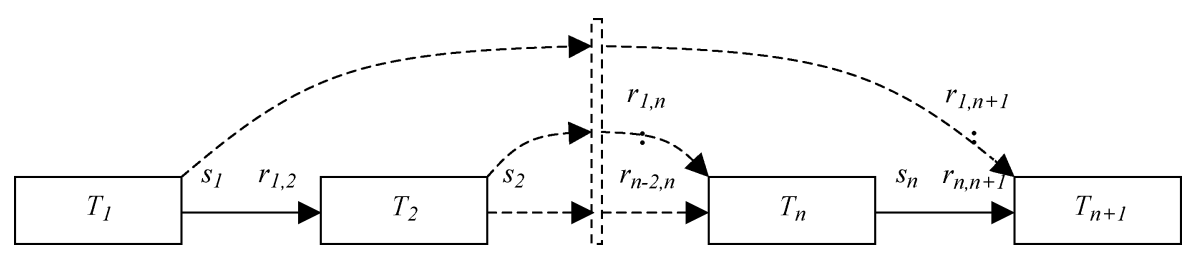

Fig. 1. Multihop wireless communications channel.

ately preceding terminal before retransmission. The amplified relaying multihop channel corresponds to the case where each intermediate terminal simply amplifies the received signal from the immediately preceding terminal before retransmission. The decoded relaying multihop diversity channel corresponds to the case where each intermediate terminal combines, digitally decodes, and re-encodes the received signals from all preceding terminals before retransmission. The amplified relaying multihop diversity channel corresponds to the case where each intermediate terminal simply combines and amplifies the received signals from all preceding terminals before retransmission.

These channel models are described via a set of mathematical characterizations that provide comparison with the traditional "singlehop" (direct transmission) channel model used as a base reference. Specific attributes compared include signal-to-noise ratio (SNR), probability of outage, probability of error, and optimal power allocation. Simulations are executed in order to validate the theoretical development and analyze the peak performance and sensitivity to intermediate terminal placement of each channel model.

\section{SySTEM MODEL}

The scope of this paper is limited to a single-user communication in isolation. Therefore, the multiple-access interference (MAI) created by the signals of other concurrent users is ignored in the analytical results. Even so, the nature of multihop relaying channels creates some unique channel-allocation questions. Current literature [10], [19] on the subject of multihop wireless communications channels places the restriction that relaying must be performed in a separate channel, due to limitations in the signal-processing capabilities of terminal hardware. The concern is that using the same channel will result in feedback from the transmitter to the receiver, and since the transmitted power is generally many orders of magnitude greater than the received power, the received signal may be completely obscured. Relaying terminals are then forced to allocate at least one separate channel for relaying, and therefore, use more system resources per communications link than the singlehop reference channel. Also, depending on the specific multiple-access scheme chosen, additional channels may be required. For example, a time-division multiple-access (TDMA) scheme would generally involve each relay retransmitting the signal in a new time slot, therefore requiring a separate channel for each transmitter in the multihop transmission path.

The issue of channel and resource allocation for multihop networks is a very rich and interesting field that cannot be addressed fully in the context of this paper. Therefore, the scope has been limited to the physical level with minimal constraints imposed on higher-level allocation schemes. For the purpose of this paper, it is sufficient to assume that orthogonal channels are achieved through some combination of time, frequency, and code-division multiple access (CDMA) as part of an overall channel- and resource-allocation scheme. Discussion related to the problem of selecting channels for relaying in cellular networks is presented in [29].

The system model for multihop wireless communications channels is composed of a source terminal, a destination terminal, and a variable number of intermediate relaying terminals. In Fig. 1, the source terminal is identified as $T_{1}$, the destination terminal is identified as $T_{n+1}$, and the intermediate terminals are identified as $T_{2}-T_{n}$, where $n$ is the number of hops along the transmission path. The only difference between multihop channels with and without diversity is the signal connectivity between nonadjacent terminals. Whereas terminals participating in multihop channels without diversity process only the signal from the immediately preceding terminal, terminals participating in multihop channels with diversity combine and process the signals from all preceding terminals.

Let $T_{S}$ represent the set of source terminals, $T_{I}$ represent the set of intermediate terminals, and $T_{D}$ represent the set of destination terminals. Therefore, $T_{T}=T_{S} \cup T_{I}$ represents the set of all transmitting terminals, and $T_{R}=T_{I} \cup T_{D}$ represents the set of all receiving terminals. Let $T_{P(i)}$ represent the set of terminals that transmit a signal received by terminal $T_{i}$. The notation used in this paper assumes that $T_{P(i)}$ has cardinality equal to one for channels without diversity, and greater than or equal to one for channels with diversity. For the channel illustrated in Fig. $1, T_{S}=\left\{T_{1}\right\}, T_{I}=\left\{T_{2}, \ldots, T_{n}\right\}, T_{D}=\left\{T_{n 1}\right\}$ $T_{T}=\left\{T_{1}, \ldots, T_{n}\right\}, T_{R}=\left\{T_{2}, \ldots, T_{n+1}\right\}$, and (for multihop channels without diversity) $T_{P(i)}=\left\{T_{i-1}\right\}, 2 \leq i \leq n+1$ or (for multihop channels with diversity) $T_{P(i)}=\left\{T_{1}, \ldots, T_{i-1}\right\}$, $2 \leq i \leq n+1$. These definitions are used in variable subscripts to denote specific terminals or sets of terminals. Notation of the form $x_{T_{i}}$ is abbreviated to $x_{i}$ for simplicity of exposition.

Each terminal $T_{i}$ transmits a discrete-time signal with complex baseband amplitude given by

$$
s_{i}=\sqrt{\varepsilon_{i}}\left(\alpha_{i}+\beta_{i}\right)
$$

where $\varepsilon_{i}$ is the transmitted power, $\alpha_{i}$ is the complex amplitude of the information symbol during a particular signaling interval, and $\beta_{i}$ is propagated noise. It is important to note that this model normalizes the information and noise portions of the transmitted signal, such that $\left|\alpha_{i}\right|^{2}+E\left[\left|\beta_{i}\right|^{2}\right]=1$. Since the only terminals that propagate noise are intermediate terminals that employ amplified relaying, for all other terminals (source terminals and intermediate terminals that employ decoded relaying), $\beta_{i}=0$ and the above normalization simplifies to $\left|\alpha_{i}\right|^{2}=1$. The definition of $\varepsilon_{i}$ has been modified from [3] such that it is the transmitted power of both the information and propagated-noise portions of 
the transmitted signal, instead of only the information portion. The result is equations for amplified relaying channels that are more amenable to analysis and transmit-power optimization.

A simple wireless channel model with flat (frequency-nonselective) fading and shadowing is chosen in order to highlight the benefits of multihop diversity. Each interuser channel is considered to be mutually independent. Using this model, each terminal $T_{i}$ then receives a set of discrete-time signals with complex baseband amplitudes given by

$$
r_{k, i}=\left(\delta \sqrt{\left(\frac{L_{k, i}}{d_{k, i}^{p}}\right)} R_{k, i}\right) \sqrt{\varepsilon_{k}}\left(\alpha_{k}+\beta_{k}\right)+z_{k, i}, T_{k} \in T_{P(i)}
$$

where $\delta^{2}$ is the free space signal-power attenuation factor between the transmitting terminal and an arbitrary reference distance, $d_{k, i}$ is the interterminal distance normalized with respect to the reference distance, $p$ is the propagation exponent, $L_{k, i}$ is a lognormal random variable with mean $0 \mathrm{~dB}$ and variance $\sigma_{L_{k, i}}^{2}, R_{k, i}$ is a complex Gaussian (Rayleigh) random variable with variance $\sigma_{R_{k, i}}^{2}$, and $z_{k, i}$ is a zero-mean Gaussian random variable with variance $N_{k, i}$.

Using this model, the received SNR at $T_{i}$ for the signal from each preceding terminal $T_{k}$ is given by

$$
\gamma_{k, i}=\frac{\varepsilon_{k}\left|\alpha_{k}\right|^{2}}{\left(\frac{d_{k, i}^{p}}{\delta^{2} L_{k, i}\left|R_{k, i}\right|^{2}}\right) N_{k, i}+\varepsilon_{k} E\left[\left|\beta_{k}\right|^{2}\right]}, \quad T_{k} \in T_{P(i)}
$$

where $\left|R_{k, i}\right|^{2}$ is an exponential random variable with mean $2 \sigma_{R_{k, i}}^{2}$.

The probability of outage due to lognormal shadowing when $\beta_{k}=0$ is given according to the method in [25] by

$$
\operatorname{Pr}\left[\widetilde{\gamma}_{k, i}<\gamma\right] \approx Q\left(\frac{10 \log \left(\frac{\overline{\gamma_{k, i}}}{\gamma}\right)}{\sigma_{L_{k, i}}}\right), \quad T_{k} \in T_{P(i)}
$$

where $\widetilde{\gamma}_{k, i}$ is the instantaneous received SNR at $T_{i}$ averaged over the Rayleigh fading, $\overline{\gamma_{k, i}}$ is the expected received SNR at $T_{i}$ averaged over the lognormal shadowing and Rayleigh fading, $\gamma$ is an arbitrary threshold SNR that must be maintained at every decoder in order to maintain communication, and $Q(x)=(1 / 2) \operatorname{erfc}(x / \sqrt{2})$.

The calculation of probability of error is dependent on the modulation scheme employed. For the special case of binary phase-shift keying (BPSK), the probability of error averaged over Rayleigh fading when $\beta_{k}=0$ is given according to the method in [23] by

$$
P_{e}\left(\gamma_{k, i}\right)=\frac{1}{2}\left(1-\sqrt{\frac{\overline{\gamma_{k, i}}}{2+\overline{\gamma_{k, i}}}}\right), \quad T_{k} \in T_{P(i)}
$$

The results (4) and (5) describe the probably of outage and error performance of each individual hop, exclusive of noise propagation, within a composite multihop-relaying network. The next sections derive closed-form results for the end-to-end probability of outage and error of multihop channels employing decoding relaying and amplified relaying, respectively.

\section{DECODED RELAYING}

The channel model for the decoded relaying channels is composed of a set of individual transmission channels characterized by (1)-(3) with $\beta_{k}=0, T_{k} \in T_{P(i)}$. For decoded relaying, it is assumed that an outage or error event at any intermediate terminal along the multihop transmission path will result in a corresponding outage or error event at the destination terminal, due to propagation of the outage or error event. Although the equations resulting from this assumption are not exact, they provide a tight upper bound on the total (end-to-end) probability of outage and error, when such outage and errors events occur with low probability $(\ll 1)$. Exact equations for the probability of outage and error are derived in [3]. When all preceding terminals along the transmission path have decoded correctly, the signals from these terminals can be combined using maximal ratio combining. The received SNR at terminal $T_{i}$ is then given by

$$
\gamma_{P(i), i}=\sum_{T_{k} \in T_{P(i)}}\left(\frac{\varepsilon_{k}\left|\alpha_{k}\right|^{2}}{\left(\frac{d_{k, i}^{p}}{\delta^{2} L_{k, i}\left|R_{k, i}\right|^{2}}\right) N_{k, i}}\right)
$$

The total probability of outage for the decoded relaying channels is given according to the method in [25] by

$$
P_{o} \leq 1-\prod_{T_{i} \in T_{R}}\left(1-Q\left(\frac{10 \log \left(\frac{\overline{\gamma_{P(i), i}}}{\gamma}\right)}{\sigma_{L_{Z(i)}}}\right)\right)
$$

where $\sigma_{L_{Z(i)}}^{2}$ is the variance of the lognormal approximation of (6) determined using Wilkinson's method [1]. This method approximates the power sum of a set of lognormal random variables with another lognormal random variable. For the approximation, each lognormal component $L_{k, i}$ is independent with mean $\mu_{L_{k, i}}=\ln \left(\varepsilon_{k}\left|\alpha_{k}\right|^{2} \delta^{2} / d_{k, i}^{p}\right)$ and standard deviation $\sigma_{L_{k, i}}=\Omega$, where $\Omega$ is typically between 6-12 dB. The inequality results from the previously stated assumption regarding outage propagation. Applying the union bound, this value can be upper bounded by

$$
P_{o} \leq \sum_{T_{i} \in T_{R}} Q\left(\frac{10 \log \left(\frac{\overline{\gamma_{P(i), i}}}{\gamma}\right)}{\sigma_{L_{Z(i)}}}\right)
$$

The total probability of decoding error for the decoded relaying channels is given according to the method in [23] by

$$
\begin{aligned}
& P_{e} \leq 1-\prod_{T_{i} \in T_{R}}\left(1-\frac{1}{2}\left(\sum _ { T _ { k } \in T _ { P ( i ) } } \left[\prod_{\substack{T_{j} \in T_{P(i)} \\
T_{j} \neq T_{k}}} \frac{\overline{\gamma_{k, i}}}{\overline{\gamma_{k, i}}-\overline{\gamma_{j, i}}}\right.\right.\right. \\
& \left.\left.\times\left(1-\sqrt{\frac{\overline{\gamma_{k, i}}}{2+\overline{\gamma_{k, i}}}}\right)\right]\right)
\end{aligned}
$$

where $\overline{\gamma_{k, i}}$ is the expected received SNR at $T_{i}$ for the branch of the diversity combiner associated with the signal incident from 
terminal $T_{k}$. Once again, the inequality results from the previously stated assumption regarding error propagation. Applying the union bound, this value can be upper bounded by

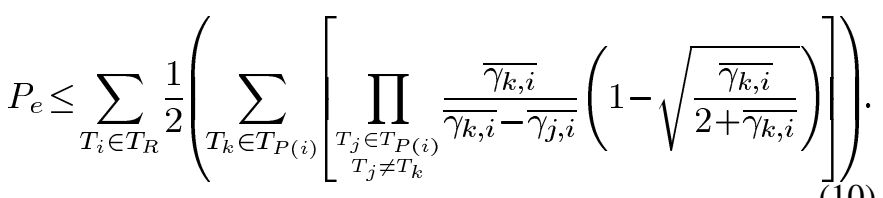

In order to provide a fair comparison with the reference channel, the transmit powers at each terminal are constrained so that the sum of the powers at each hop is equal to the reference power $\varepsilon_{0}$, namely, $\varepsilon_{0}=\sum_{T_{i} \in T_{T}} \varepsilon_{i}$. The upper bound of the total probability of decoding error for the decoded relaying channels is minimized when the sum of the probabilities of decoding error at each terminal in (10) is minimized. For the decoded relaying multihop channel, when all noise terms are statistically identical, $N_{k, i}=N_{0}, \forall N_{k, i}$, the optimal power allocation based on the upper bound in (10) is approximated by

$$
\varepsilon_{P(i)} \approx \frac{\varepsilon_{0} \sqrt{\frac{d_{P(i), i}^{p}}{L_{P(i), i}}}}{\sum_{T_{j} \in T_{R}} \sqrt{\frac{d_{P(j), j}^{p}}{L_{P(j), j}}}} .
$$

For the proof of (11), refer to Appendix A.

For the decoded relaying multihop diversity channel, the solution (even approximate) to the optimal power-allocation minimization problem is not as tractable. Although (10) is convex with respect to the transmit powers at each terminal, and the solution for any finite number of hops can be calculated using Lagrange multipliers, the resulting equations are extremely complex and difficult to generalize.

\section{AMPLIFIED RELAYING}

The channel model for the amplified relaying channels is composed of a set of individual transmission channels characterized by (1)-(3). Assuming that each receiving terminal can track Rayleigh fading, the amplification factor at each intermediate terminal $T_{i}$ is simply the transmitted power over the received power and is given by

$$
A_{i}=\frac{\varepsilon_{i}}{\sum_{T_{k} \in T_{P(i)}}\left(\left(\frac{\delta^{2} L_{k, i}\left|R_{k, i}\right|^{2}}{d_{k, i}^{p}}\right) \varepsilon_{k}+N_{k, i}\right)} .
$$

Note that the concept of an amplification factor is irrelevant for decoded relaying, since the process of decoding removes the necessity for analog amplification.

In the case of the amplified relaying multihop diversity channel, it is important to note that the propagated noise from multiple preceding terminals along the transmission path is not mutually independent, but is, in fact, correlated. Consider a simple multihop communication channel consisting of terminals $T_{1}, T_{2}, T_{3}$, and $T_{4}$. Each of the receiving terminals $T_{2}$, $T_{3}$, and $T_{4}$ have mutually independent additive noise. Since the additive noise at terminal $T_{2}$ is propagated to terminal $T_{3}$ for retransmission, the propagated noise from terminals $T_{2}$ and $T_{3}$ received at terminal $T_{4}$ will be correlated. Although the derivation of the optimal combiner that leverages this correlation and resultant output SNR are beyond the scope of this paper, the output SNR of the optimal combiner for a generalized amplified relaying multihop diversity channel can be lower bounded in the following fashion. It is well known that the lowest output SNR of an optimal combiner occurs when the noise components of the signals received at all the input branches are mutually independent [26]. It is also well known that the optimal combiner for a set of input branches that are mutually independent is a maximal ratio combiner [26]. Therefore, considering the worst possible case, when the propagated noise from all preceding terminals is mutually independent, the average received SNR at each received terminal can be lower bounded by the performance of a maximal ratio combiner.

The received SNR at the destination terminal $T_{d}$ is then expressible recursively as

$$
\gamma_{P(d), d}=\sum_{\substack{T_{k} \in T_{P}(d) \\ k \neq s}}\left(\gamma_{P(k), k}^{-1}+\psi_{k, d}^{-1}+\gamma_{P(k), k}^{-1} \psi_{k, d}^{-1}\right)^{-1}+\psi_{s, d}
$$

where $\psi_{k, d}$ is the received SNR, $\gamma_{k, d}$, with $B_{k}=0$ at terminal $T_{d}$ for the branch of the diversity combiner associated with the signal incident from terminal $T_{k}$, and $T_{s}$ is the source terminal. For amplified relaying channels without diversity, there is only one term in (13), since only the signal of the immediately preceding terminal along the transmission path is received. For the first receiving terminal, the only term is $\psi_{s, d}$ with $\sum_{\substack{T_{k} \in T_{P(d)} \\ k \neq s}}\left(\gamma_{P(k), k}^{-1}+\psi_{k, d}^{-1}+\gamma_{P(k), k}^{-1} \psi_{k, d}^{-1}\right)^{-1}=0$, and for the remaining receiving terminals, the only term is $\left(\gamma_{P(k), k}^{-1}+\right.$ $\left.\psi_{k, d}^{-1}+\gamma_{P(k), k}^{-1} \psi_{k, d}^{-1}\right)^{-1}, T_{k}=T_{P(d)}$ with $\psi_{s, d}=0$. For the proof of (13), refer to Appendix B. Also note that when there are only two hops, (13) reduces to the results for amplify-and-forward transmission presented in [20] and [22].

The probability of outage for the amplified relaying channels at the destination terminal is given by

$$
P_{o}=\operatorname{Pr}\left[\widetilde{\gamma}_{P(d), d}<\gamma\right] \approx Q\left(\frac{10 \log \left(\frac{\overline{\gamma_{P(d), d}}}{\gamma}\right)}{\sigma_{L_{Z(d)}}}\right)
$$

where $\operatorname{Pr}\left[\widetilde{\gamma}_{P(d), d}<\gamma\right]$ is the probability of outage at the destination terminal, given a received SNR of $\gamma_{P(d), d}$, and $\sigma_{L_{Z(d)}}^{2}$ is the variance of the lognormal approximation of (13) determined using Wilkinson's method [1]. Alternative methods for characterizing the outage statistics of multihop channels are studied in [11] and [31].

The total probability of decoding error for the amplified relaying channels is given by

$$
\begin{aligned}
P_{e} & =P_{e}\left(\gamma_{P(d), d}\right) \\
& =\frac{1}{2}\left(\sum_{T_{k} \in T_{P(d)}}\left[\prod_{\substack{T_{j} \in T_{P(d)} \\
T_{j} \neq T_{k}}} \frac{\overline{\gamma_{k, d}}-\overline{\gamma_{j, d}}}{\overline{\gamma_{j, d}}}\left(1-\sqrt{\frac{\overline{\gamma_{k, d}}}{2+\overline{\gamma_{k, d}}}}\right)\right]\right)
\end{aligned}
$$

where $P_{e}\left(\gamma_{P(d), d}\right)$ is the probability of decoding error at the destination terminal, given a received SNR of $\gamma_{P(d), d}$, and $\overline{\gamma_{k, d}}$ is the expected received SNR at the destination terminal for the branch of the diversity combiner associated with the signal incident from terminal $T_{k}$. 
As for the decoded relaying channels, in order to provide a fair comparison with the reference channel, the transmit powers at each terminal are constrained so that the sum of the powers at each hop is equal to the reference power $\varepsilon_{0}$, namely, $\varepsilon_{0}=$ $\sum_{T_{i} \in T_{T}} \varepsilon_{i}$. The total probability of decoding error for the amplified relaying channels is minimized when the received SNR at the destination terminal is maximized. For the amplified relaying multihop channel when all noise terms are statistically identical, $N_{k, i}=N_{0}, \forall N_{k, i}$, the optimal power allocation based on (13) is approximated by

$$
\varepsilon_{P(i)} \approx \frac{\varepsilon_{0} \sqrt{\frac{d_{P(i), i}^{p}}{L_{P(i), i}}}}{\sum_{T_{j} \in T_{R}} \sqrt{\frac{d_{P(j), j}^{p}}{L_{P(j), j}}}} .
$$

For the proof of (16), refer to the proof of (11) in Appendix A.

The determination of the optimal power allocation for the amplified relaying multihop diversity channel is of the same form and complexity as the decoded relaying multihop diversity channel.

\section{FEEDBACK AND FEEDFORWARD INTERFERENCE}

The primary metric used thus far to characterize the channel models is the SNR, as opposed to the signal-to-interferenceplus-noise ratio (SINR). The motivation for this is that the scope of consideration is a single-user channel in isolation, allowing MAI to be ignored. However, there are two forms of interference that are unique to multihop channels. These are denoted feedback interference and feedforward interference, and are a result of interference between the signals transmitted at different hops along a multihop path. These forms of interference can also be referred to collectively as self-interference or interhop interference. From this point forward, the term SINR shall be used such that it refers only to this interhop interference, and not MAI.

Feedback interference is caused by the reception of relayed signals from following terminals along the transmission route, and affects both multihop and multihop diversity channels. Feedforward interference is caused by the reception of relayed signals from preceding terminals along the transmission route other than the immediately preceding terminal, and affects only multihop channels without diversity. These same transmitted signals, if processed and combined properly, provide the diversity in the multihop diversity channels. It is important to note that both feedback and feedforward interference are special cases of intersymbol interference (ISI), and can therefore be mitigated through traditional equalization techniques.

The feedback interference power at terminal $T_{i}$ is given by

$$
I_{V(i), i}=\frac{1}{C} \sum_{T_{j} \in T_{V(i)}}\left(\frac{\varepsilon_{j}}{\frac{d_{j, i}^{p}}{\delta^{2} L_{j, i}\left|R_{j, i}\right|^{2}}}\right)
$$

where $C$ is the processing gain of the system, and $T_{V(i)}$ represents the set of transmitting terminals following terminal $T_{i}$ along the transmission route. The definition of the system processing gain $C$ for specific multiple access and relaying schemes is discussed later in this section. The feedforward interference power at terminal $T_{i}$ is given by

$$
I_{U(i), i}=\frac{1}{C} \sum_{T_{j} \in T_{U(i)}}\left(\frac{\varepsilon_{j}}{\frac{d_{j, i}^{p}}{\delta^{2} L_{j, i}\left|R_{j, i}\right|^{2}}}\right)
$$

where $T_{U(i)}$ represents the set of transmitting terminals preceding terminal $T_{i}$ along the transmission route that do not belong to $T_{P(i)}$. Therefore, the set of all terminals along the transmission route can be represented by $T_{A}=T_{U(i)} \cup T_{P(i)} \cup T_{i} \cup$ $T_{V(i)} \cup T_{D}$.

Of interest is the impact of this feedback and feedforward interference on the performance of the multihop channels. Let us consider the worst-case scenario, where the combined ISI is not mitigated in any way. For the decoded relaying channels, it follows from (6) that the received SINR at terminal $T_{i}$ is given by

$\mathrm{H}_{P(i), i}=\sum_{T_{k} \in T_{P(i)}}\left(\frac{\varepsilon_{k}\left|\alpha_{k}\right|^{2}}{\left(\frac{d_{k, i}^{p}}{\delta^{2} L_{k, i}\left|R_{k, i}\right|^{2}}\right)\left(N_{0}+I_{U(i), i}+I_{V(i), i}\right)}\right)$.

For the amplified relaying channels, it follows from (13) that the received SINR at the destination terminal $T_{d}$ can be expressed recursively as

$$
\mathrm{H}_{P(d), d} \approx \sum_{\substack{T_{k} \in T_{P(d)} \\ k \neq s}}\left(\mathrm{H}_{P(k), k}^{-1}+\Psi_{k, d}^{-1}+\mathrm{H}_{P(k), k}^{-1} \Psi_{k, d}^{-1}\right)^{-1}+\Psi_{s, d}
$$

where $\Psi_{k, d}$ is the received SINR, $\mathrm{H}_{k, d}$, with $\beta_{k}=0$ at terminal $T_{d}$ for the branch of the diversity combiner associated with the signal incident from terminal $T_{k}$, and is given by

$$
\Psi_{k, d}=\frac{\varepsilon_{k}\left|\alpha_{k}\right|^{2}}{\left(\frac{d_{k, d}^{p}}{\delta^{2} L_{k, d}\left|R_{k, d}\right|^{2}}\right)\left(N_{0}+I_{U(d), d}+I_{V(d), d}\right)}
$$

and $T_{s}$ is the source terminal.

Of particular importance in the preceding equations is the determination of the processing gain $C$. The processing gain of the system is dependent on the chosen multiple access and relaying scheme. For CDMA systems, $C$ is equal to the number of chips per bit (spreading gain). For TDMA and frequencydivision multiple-access (FDMA) (or orthogonal (O)FDMA) systems that use the same channel for relaying as the source transmission, $C$ is equal to unity and the interference is a delayed and distorted version of the desired signal. For TDMA and FDMA (or OFDMA) systems that use a separate channel for relaying, as well as contention-based multiple-access systems, $C$ approaches infinity and is limited only by adjacent channel interference.

\section{Simulation RESUlts}

The results presented thus far are applied in two simulations that provide a comparison with the singlehop reference channel on the basis of probability of error. A BPSK modulation scheme is used for simplicity of exposition, with relaying in a separate orthogonal channel. The feedback and feedforward interference 


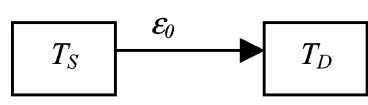

Reference Channel

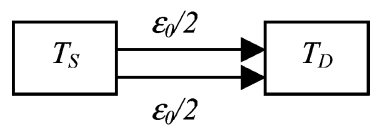

2x Reference Channel

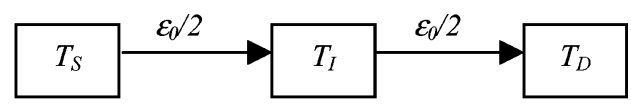

Multihop Channel (2 hops)

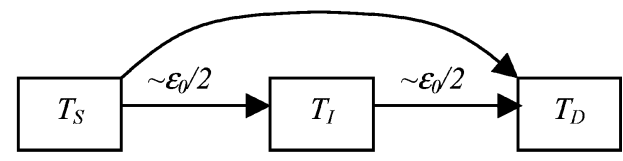

Multihop Diversity Channel (2 hops)

Fig. 2. Example simulated channels indicating transmit power.

presented in the last section is assumed to be negligible. A maximal ratio combiner is assumed at receiving terminals for multihop channels with diversity. The coordinates of the channel are normalized with respect to the distance between the source $T_{1}$ and destination $T_{n+1}$, such that $d_{1, n+1}=1$. The propagation exponent is $p=4$. For the purpose of simplifying the comparison, and without loss of generality, the free-space signal-attenuation factor is $\delta^{2}=1$. The variance of all Rayleigh random variables is $\sigma_{R_{k, i}}^{2}=0.5$, such that the mean of the corresponding exponential random variables is $2 \sigma_{R_{k, i}}^{2}=1$. The optimal power allocation is assumed for each of the channel models, with total power constrained to the reference power. Outage results for multihop wireless communications channels are presented in [3].

\section{A. Fixed Intermediate Terminal Positions With n Hops}

For the first set of simulations, the intermediate terminals are fixed and collinear, so that they divide the direct path between the source and destination terminals into equal-length segments. This serves to validate the theoretical derivations presented thus far, as well as illustrate the benefit that can be realized under an optimal placement of intermediate terminals with respect to the source and destination terminals. The graphs provide a comparison in terms of probability of error versus the SNR of the singlehop reference channel. The probability of error results for the multihop channels are plotted such that the total allocated power of each multihop channel is the same as that of the singlehop reference channel that achieves the indicated SNR.

Also simulated is the $2 \times$ reference channel, wherein direct communication occurs between the source and destination terminals over two separate orthogonal channels in parallel. Each orthogonal channel uses one half of the reference power, such that the total power is constrained to the reference power. The separate orthogonal channels are assumed to experience mutually independent fading (such as when each channel is at a different frequency). The resultant mutually independent fading signals are then combined coherently at the destination terminal in order to calculate the total probability of decoding error. The $2 \times$ reference channel therefore experiences diversity gain of order two under a total power constraint. This provides a fair reference for comparison in terms of channel use with multihop channels that require a separate orthogonal channel for relaying. Fig. 2 shows an example of the four classes of simulated channels, including the transmitted power of each terminal.

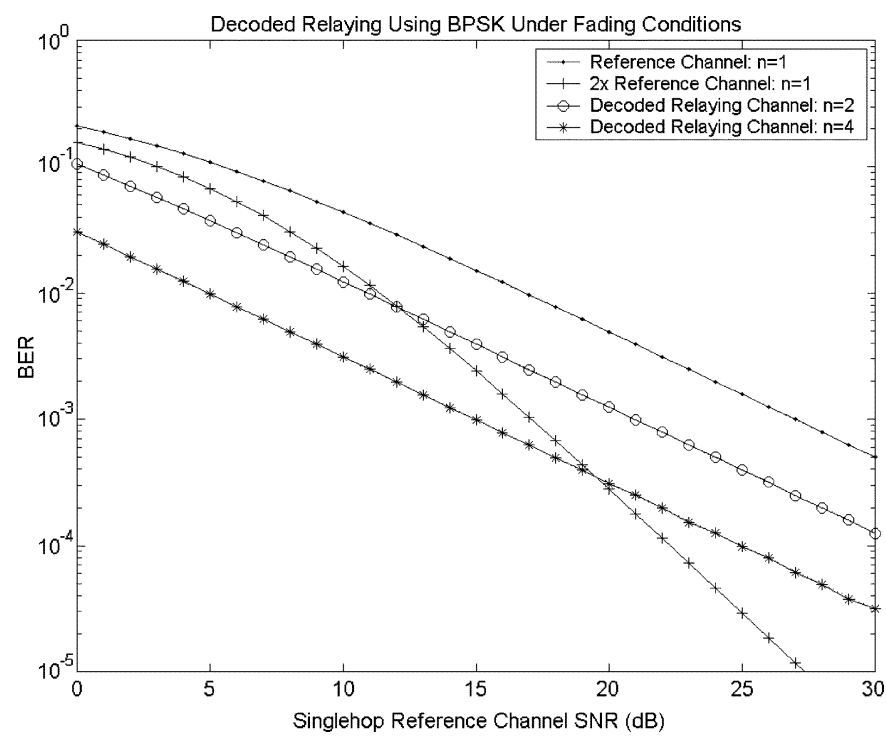

Fig. 3. Probability of error for decoded relaying multihop channel.

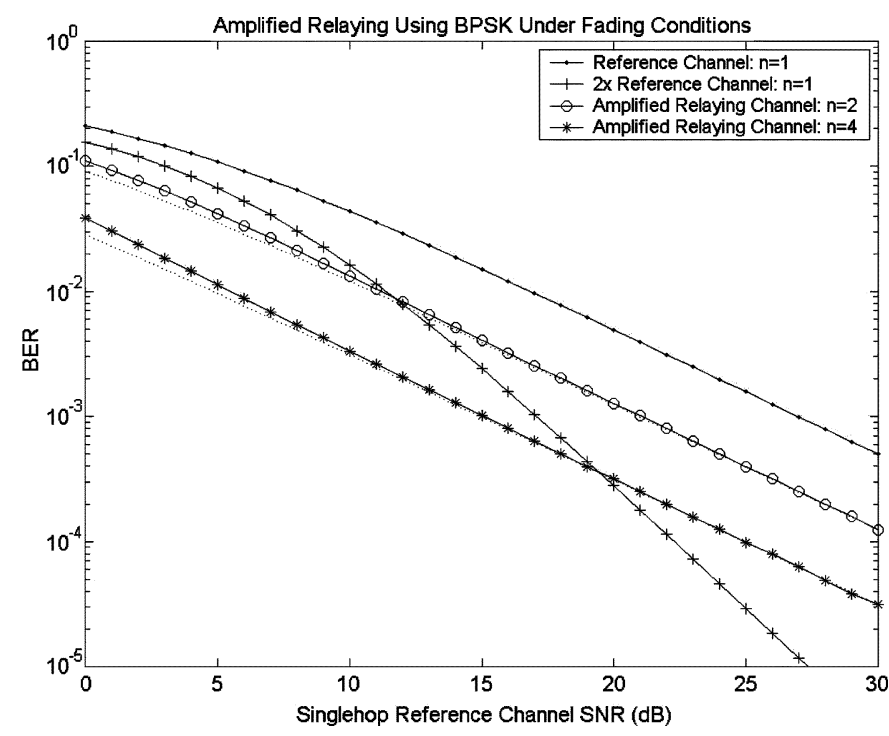

Fig. 4. Probability of error for amplified relaying multihop channel.

Figs. 3-6 show the simulated probability of error performance under collinear intermediate terminal placement. The theoretical characterizations (10) and (15) are represented by dotted lines, and the simulated results are represented by solid 


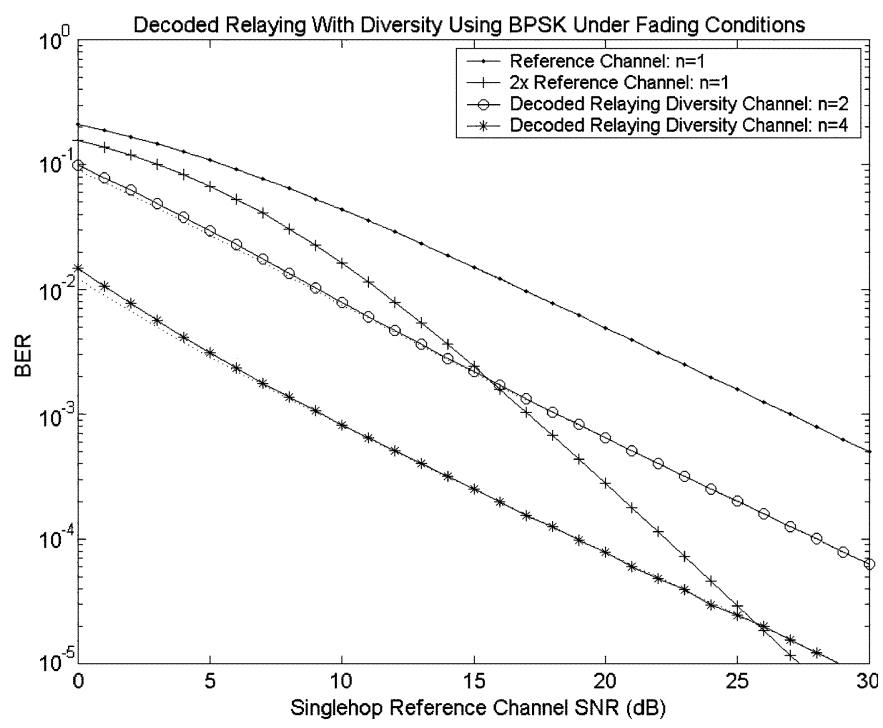

Fig. 5. Probability of error for decoded relaying multihop diversity channel.

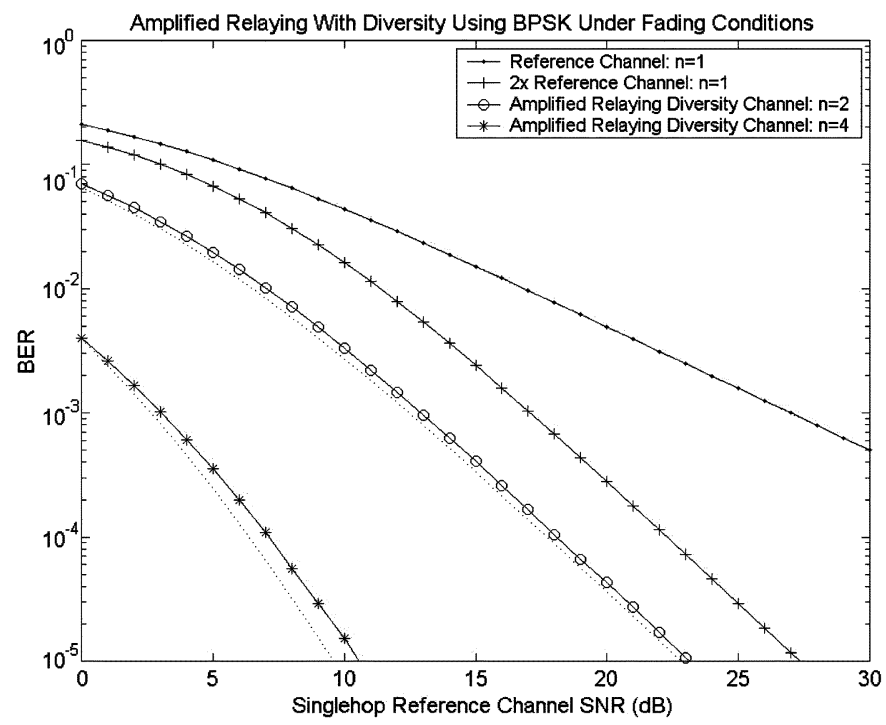

Fig. 6. Probability of error for amplified relaying multihop diversity channel.

lines. Note that the theoretical and simulated results for the decoded relaying channels use the approximation that a decoding error at any intermediate terminal will result in a decoding error at the destination terminal.

The graphs indicate that the multihop channels with diversity significantly outperform the multihop channels without diversity, and that the amplified relaying channels experience performance gains that are equal to or greater than the decoded relaying channels. The performance results of the decoded relaying multihop channel and the amplified relaying multihop channel are similar. The performance results of the amplified relaying multihop diversity channel are better than those of the decoded relaying multihop diversity channel. An explanation for this result lies in the structure of the decoded relaying receiver implied by (9) and (10). Whereas the amplified relaying channel responds gracefully to severe signal degradation on any individual link between two terminals, the decoded relaying channel produces an error at the destination receiver if any individual link between two terminals produces an error. This is a result of the propagation of errors to following terminals along the transmission path, significantly decreasing the error performance. Thus, the amplified relaying multihop diversity channel is the only multihop model that makes full use of the inherent diversity available in multihop relaying networks. It is also important to note that other relaying protocols have been proposed that improve the performance of decoded relaying [12], [20]-[22]. These involve coding techniques, out-of-band feedback indicating success of reception, and adaptive behavior based on receiver channel information. Many of these techniques can also be applied to amplified relaying with similar performance improvement [21], [22].

As expected, the multihop channels all outperform the singlehop reference channel and the $2 \times$ reference channel at low SNRs. However, as the SNR is increased, it is shown that the $2 \times$ reference channel eventually outperforms all the multihop channels except for the amplified relaying multihop diversity channel. This results directly from the fact that the amplified relaying multihop diversity channel is the only multihop model that achieves full diversity benefits.

The difference between the theoretical and simulated results is due to the receiver model used in the simulations. A maximal ratio combiner is used, even though in the simulations, the propagated noise from some preceding terminals is correlated, as would occur in practice. Thus, in the simulations, the receiver model is suboptimal, resulting in the simulated performance being worse than the theoretical performance. The difference between the theoretical and simulated performance results therefore illustrates the performance degradation of the maximal ratio combiner due to correlation of the incident-propagated noise.

Note that the use of optimal power allocation assumes that the attenuation and shadowing characteristics at each relay terminal are known. In practice, optimal allocation may be difficult to achieve in a distributed fashion. Various publications address issues relevant to power control in distributed networks [7]-[9], [30], [32].

\section{B. Variable Intermediate Terminal Position With Two Hops}

For the second set of simulations, a single intermediate terminal is placed at locations uniformly distributed across a unit square. This serves to illustrate the robustness of the channel models with respect to distance from the optimal placement of the intermediate terminal. The graphs provide a comparison in terms of probability of error versus intermediate terminal location when the normalized average SNR is fixed at $10 \mathrm{~dB}$. Figs. 7-10 show the variation of the error performance with respect to the position of the intermediate terminal. The concave hulls represent the theoretical characterizations, and the horizontal plane indicates the error performance of the singlehop reference channel. The graphs indicate that the performance gain with respect to the reference channel is fairly sensitive to 


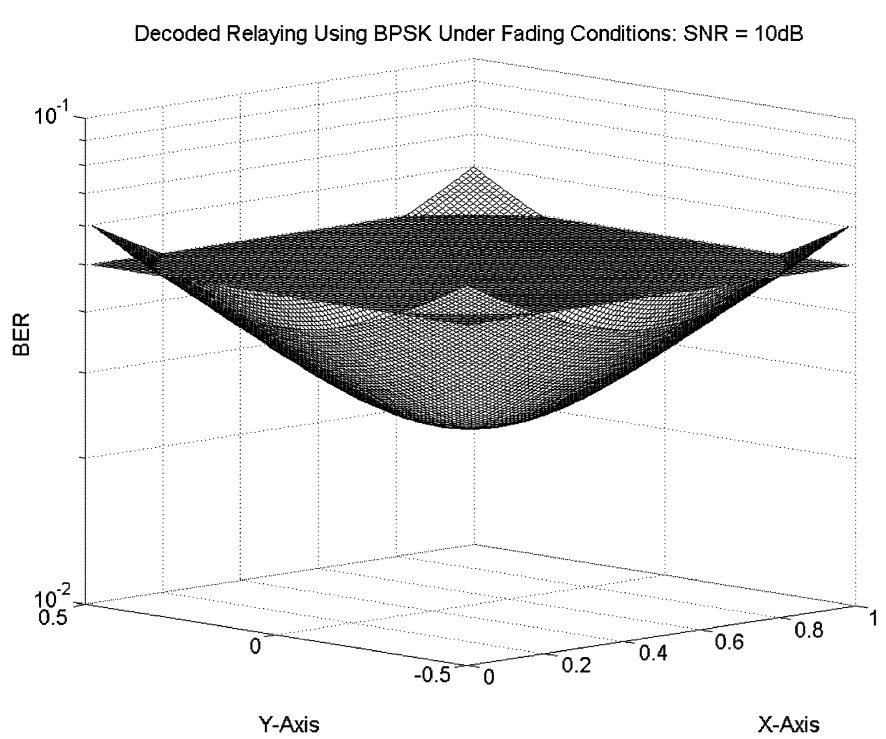

Fig. 7. Robustness of decoded relaying multihop channel.

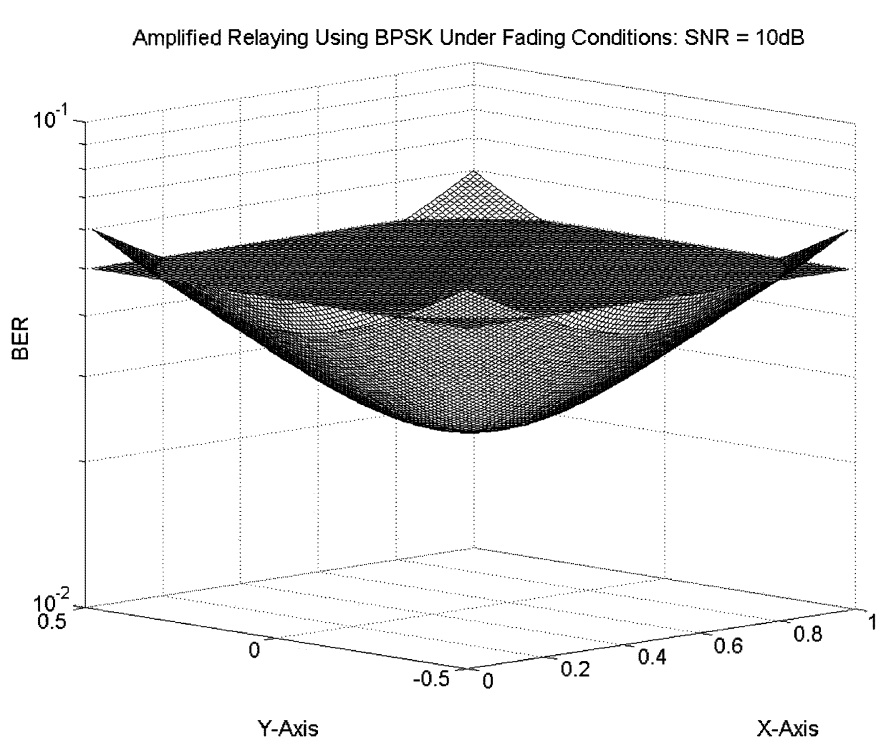

Fig. 8. Robustness of amplified relaying multihop channel.

the relative position of the intermediate terminal. The multihop diversity channels, especially with amplified relaying, are less sensitive to intermediate terminal position, in that they achieve a performance gain over the singlehop reference channel for a larger range of intermediate terminal positions.

The probability of error performance of the multihop channels without diversity is symmetrical about both the axis parallel and the axis perpendicular to the line joining the source and destination terminals. This is a result of the symmetrical form of (10) and (15). The error performance is, therefore, solely a function of the hop distances, and is not dependent on the respective distances of the intermediate terminal from the source and destination terminals.

The probability of error performance of the multihop channels with diversity is symmetrical about the axis parallel to the line joining the source and destination terminals, but not
Decoded Relaying With Diversity Using BPSK Under Fading Conditions: SNR = 10dB

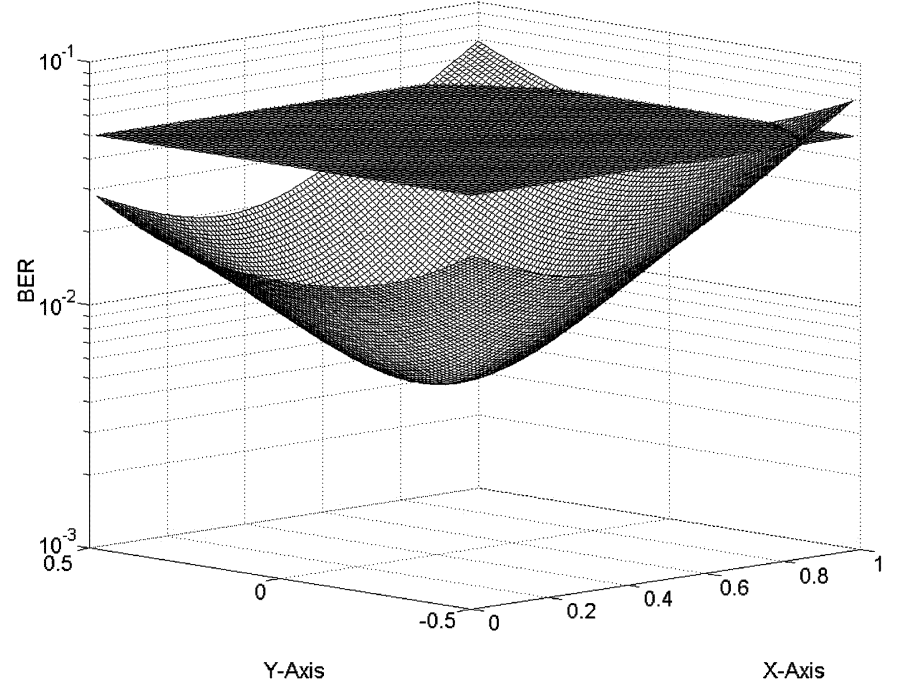

Fig. 9. Robustness of decoded relaying multihop diversity channel.

Amplified Relaying With Diversity Using BPSK Under Fading Conditions: SNR = 10dB

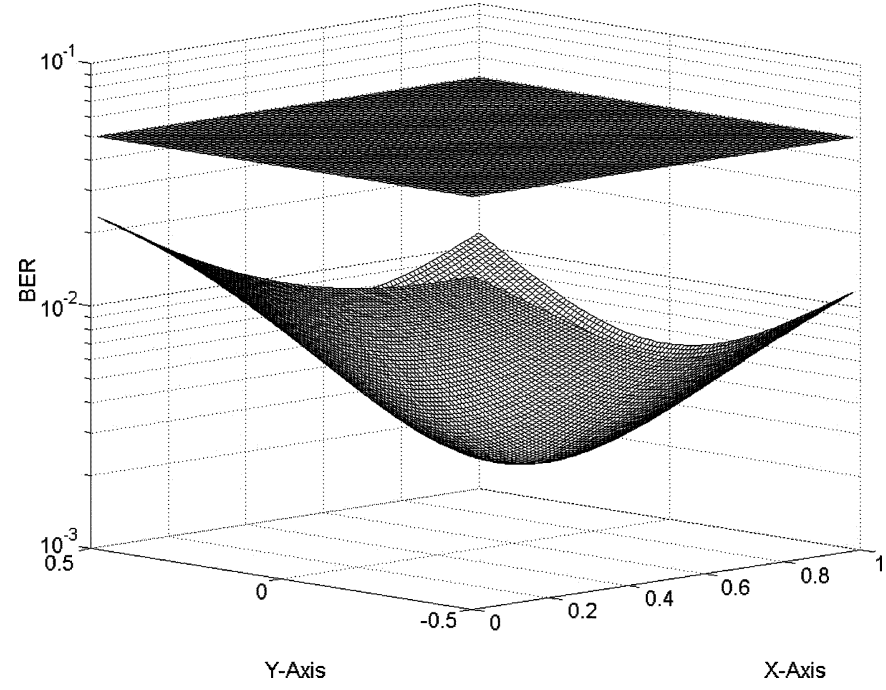

Fig. 10. Robustness of amplified relaying multihop diversity channel.

the axis perpendicular to that line. The error performance of the decoded relaying multihop diversity channel is generally better when the intermediate terminal is closer to the source terminal than the destination terminal. This is due to the fact that the limiting term in (10) is the probability of error on the hop between the source terminal and the intermediate terminal, where no benefit is gained from diversity combining. The error performance of the amplified relaying multihop diversity channel is generally better when the intermediate terminal is closer to the destination terminal than the source terminal. This is due to the fact that as the distance from the intermediate terminal to the destination terminal decreases, the intermediate terminal requires a smaller percentage of the total allocated channel power, allowing the source terminal to transmit with greater power.

These results attest to the importance of good decisions when selecting intermediate terminals. The gain indicated in the first 
set of simulations becomes negligible, and in some cases, negative, as the distance with respect to that midpoint position increases. The determination of whether a particular terminal should be used for relaying and which terminals represent the best relaying set is generally referred to as ad-hoc routing [13]. A number of different ad-hoc routing protocols have been proposed [4], [5], [15], [16] that use a variety of methods to cope with the frequent changes in network topology caused by the combination of terminal mobility and multipath fading.

\section{CONCLUSION}

Four channel models for multihop wireless communication are proposed and characterized. Simulations indicate that significant performance improvements can be realized through the use of multihop channels, but that these performance improvements are fairly sensitive to the location of the intermediate terminals under a total power constraint. All four multihop channels are shown to outperform the singlehop reference channel, provided that relaying terminals are chosen intelligently, with the multihop channels with diversity outperforming the multihop channels without diversity. The performance of amplified relaying, especially amplified relaying with multihop diversity, is shown to be generally better than that of decoded relaying, despite noise propagation. This important and somewhat unexpected result is due to the fact that amplified relaying channels do not suffer from the weakest-link limitation affecting decoded relaying channels, wherein decoding errors on any single hop are propagated along the channel. All four multihop channel models outperform the $2 \times$ reference channel at low SNRs, while the amplified relaying multihop diversity channel outperforms the $2 \times$ reference channel even at high SNRs. The performance of the decoded relaying multihop diversity channel is generally better when the intermediate terminals are closer to the source terminal than the destination terminal, since the performance is limited by the first hop where there is no diversity benefit. The performance of the amplified relaying multihop diversity channel is generally better when the intermediate terminals are closer to the destination terminal than the source terminal, since the intermediate terminal requires a smaller percentage of the total allocated channel power, allowing the source terminal to transmit with greater power.

\section{APPENDIX A \\ PROOF OF (11)}

The optimal power allocation that minimizes the total probability of error (10) can be approximated in the following fashion using Lagrange multipliers.

1) Approximate the total probability of error (10) according to the method in [23] by

$$
\underset{T_{i} \in T_{R}}{f}\left(\varepsilon_{P(i)}\right)=\sum_{T_{i} \in T_{R}} \frac{\left(\frac{d_{P(i), i}^{p}}{\delta^{2} L_{P(i), i}}\right) N_{0}}{2 \varepsilon_{P(i)}\left|\alpha_{P(i)}\right|^{2}} .
$$

2) Let the total power constraint be given by

$$
\underset{T_{i} \in T_{R}}{g}\left(\varepsilon_{P(i)}\right)=\varepsilon_{0}-\sum_{T_{i} \in T_{R}} \varepsilon_{P(i)}=0 .
$$

3) Using Lagrange multipliers, calculate a set of partial derivatives given by

$$
\begin{aligned}
\frac{\partial}{\partial \varepsilon_{P(i)}}\left[\underset{T_{i} \in T_{R}}{f}\left(\varepsilon_{P(i)}\right)\right. & \left.+\lambda \underset{T_{i} \in T_{R}}{g}\left(\varepsilon_{P(i)}\right)\right] \\
& =-\frac{\left(\frac{d_{P(i), i}^{p}}{\delta^{2} L_{P(i), i}}\right) N_{0}}{2 \varepsilon_{P(i)}^{2}\left|\alpha_{P(i)}\right|^{2}}-\lambda, \quad \forall T_{i} \in T_{R} .
\end{aligned}
$$

4) Set the partial derivatives equal to zero and solve the resulting system of equations for $\lambda$ and $\varepsilon_{P(i)}, \forall T_{i} \in$ $T_{R}$.

This calculation results in an approximate equation for the optimal power allocation given by (11) and completes the proof.

\section{APPENDIX B \\ PROOF OF (13)}

Let $\left|a_{i-1, i}\right|^{2}=\delta^{2} L_{i-1, i}\left|R_{i-1, i}\right|^{2} / d_{i-1, i}^{p}$. Consider first an amplified relaying channel without diversity with one hop, with source terminal $T_{1}$ and destination terminal $T_{2}$. The received SNR at terminal $T_{2}$ is then given by

$$
\gamma_{1,2}=\frac{1}{\frac{N_{0}}{\varepsilon_{1}\left|a_{1,2}\right|^{2}}} .
$$

Now consider a channel with $n$ hops with source terminal $T_{1}$, intermediate terminals $T_{2}-T_{n}$, and destination terminal $T_{n+1}$. Selecting an intermediate terminal at random, the signal received by terminal $T_{k}$ is given by

$$
r_{k-1, k}=a_{k-1, k} \sqrt{\varepsilon_{k-1}}\left(\alpha_{k-1}+\beta_{k-1}\right)+z_{k-1, k}
$$

and the received SNR at terminal $T_{k}$ is given by

$$
\gamma_{k-1, k}=\frac{\left|\alpha_{k-1}\right|^{2}}{E\left[\left|\beta_{k-1}\right|^{2}\right]+\frac{N_{k-1, k}}{\left|a_{k-1, k}\right|^{2} \varepsilon_{k-1}}} .
$$

If this signal is amplified by (12), then the signal transmitted by terminal $T_{k}$ is given by

$$
\begin{aligned}
s_{k}=\left(a _ { k - 1 , k } \sqrt { \varepsilon _ { k - 1 } } \left(\alpha_{k-1}\right.\right. & \left.\left.+\beta_{k-1}\right)+z_{k-1, k}\right) \\
& \times\left(\frac{\sqrt{\varepsilon_{k}}}{\sqrt{\left|a_{k-1, k}\right|^{2} \varepsilon_{k-1}+N_{k-1, k}}}\right)
\end{aligned}
$$

the signal received by terminal $T_{k+1}$ is given by

$$
\begin{aligned}
r_{k, k+1}=a_{k, k+1}( & \left.a_{k-1, k} \sqrt{\varepsilon_{k-1}}\left(\alpha_{k-1}+\beta_{k-1}\right)+z_{k-1, k}\right) \\
& \times\left(\frac{\sqrt{\varepsilon_{k}}}{\sqrt{\left|a_{k-1, k}\right|^{2} \varepsilon_{k-1}+N_{k-1, k}}}\right)+z_{k, k+1}
\end{aligned}
$$


and the received SNR at terminal $T_{k+1}$ is given by

$$
\begin{aligned}
& y_{k, k+1}=\frac{\left|\alpha_{k-1}\right|^{2}}{\left[\begin{array}{l}
E\left[\left|\beta_{k-1}\right|^{2}\right]+\frac{N_{k-1, k}}{\left|a_{k-1, k}\right|^{2} \varepsilon_{k-1}}+ \\
\left(\frac{N_{k, k+1}}{\left|a_{k, k+1}\right|^{2} \varepsilon_{k}}\right)\left(1+\frac{N_{k-1, k}}{\left|a_{k-1, k}\right|^{2} \varepsilon_{k-1}}\right)
\end{array}\right]} \\
& 1
\end{aligned}
$$

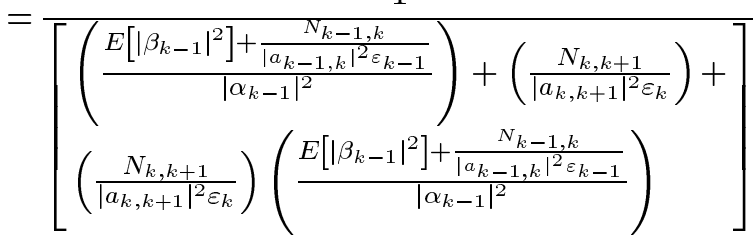

$$
\begin{aligned}
& =\frac{1}{\left[\begin{array}{l}
\left(\frac{1}{\gamma_{k-1, k}}\right)+\left(\frac{N_{k, k+1}}{\left|a_{k, k+1}\right|^{2} \varepsilon_{k}}\right)+ \\
\left(\frac{1}{\gamma_{k-1, k}}\right)\left(\frac{N_{k, k+1}}{\left|a_{k, k+1}\right|^{2} \varepsilon_{k}}\right)
\end{array}\right]} \\
& =\left(\gamma_{k-1, k}^{-1}+\psi_{k, k+1}^{-1}+\gamma_{k-1, k}^{-1} \psi_{k, k+1}^{-1}\right)^{-1} \text {. }
\end{aligned}
$$

Since it has been shown that the equation inside the summation (13) holds for the first receiving terminal, and given that if the equation holds for a particular receiving terminal, it will also hold for the next receiving terminal, the equation must hold for all receiving terminals. Using the set notation results in a generalized notation for amplified relaying channels without diversity. Given that this basic equation can be represented in inverse sum of inverses form, and that the output SNR of a maximal ratio combiner is the sum of the SNRs of the input branches [25], multihop wireless communications channels can be considered in the light of resistance theory for electrical circuits. Signal links in serial are analogous to resistors in parallel (with additional multiplicative terms). Signal links in parallel are analogous to resistors in serial. The complete network of serial and parallel signal links can then be expressed in an equivalent representation, derived in a recursive fashion. The equivalent representation of the received SNR of every terminal is, therefore, given by (13), completing the proof.

\section{REFERENCES}

[1] A. Abu-Dayya and N. Beaulieu, "Outage probabilities in the presence of correlated lognormal interferers," IEEE Trans. Veh. Technol., vol. 43, pp. 164-173, Feb. 1994.

[2] P. Anghel, G. Leus, and M. Kaveh, "Multi-user space-time coding in cooperative networks," in Proc. IEEE Int. Conf. Acoustics, Speech, Signal Processing, vol. 4, 2003, pp. 73-76.

[3] J. Boyer, "Multihop wireless communications channels," Master's thesis, Carleton Univ., Ottawa, ON, Canada, 2001.

[4] J. Broch et al., "A performance comparison of multihop wireless ad hoc network routing protocols," in Proc. IEEE/ACM MOBICOM, 1998, pp. 85-97.

[5] S. Das et al., "Comparative performance evaluation of routing protocols for mobile ad hoc networks," in Proc. Int. Conf. Computer Communications, Networks, 1998, pp. 153-161.

[6] V. Emamian, P. Anghel, and M. Kaveh, "Multi-user spatial diversity in a shadow-fading environment," in Proc. IEEE Vehicular Technology Conf., vol. 1, 2002, pp. 573-576.

[7] E. Erkip, "Capacity and power control for spatial transmit diversity," in Proc. Conf. Information Sciences, Systems, 2000, pp. WA4-28-WA4-31.

[8] G. Foschini and Z. Miljanic, "A simple distributed autonomous power control algorithm and its convergence," IEEE Trans. Veh. Technol., vol. 42, pp. 641-646, July 1993.

[9] S. Hanly, "Capacity and power control in spread spectrum macrodiversity radio networks," IEEE Trans. Commun., vol. 44, pp. 247-256, Feb. 1996.
[10] T. Harrold and A. Nix, "Intelligent relaying for future personal communications systems," in Proc. IEEE Colloq. Capacity, Range Enhancement Techniques for Third Generation Mobile Communication and Beyond, 2000, pp. 9/1-9/5

[11] M. Hasna and M. Alouini, "End-to-end performance of transmission systems with relays over Rayleigh fading channels," IEEE Trans. Wireless Commun., vol. 2, pp. 1126-1131, Nov. 2003.

[12] T. Hunter and A. Nosratinia, "Cooperation diversity through coding," in Proc. IEEE Int. Symp. Information Theory, 2002, p. 220.

[13] IEEE J. Select. Areas Commun.: Issue on Wireless Ad Hoc Networks, vol. 17, Oct. 1999

[14] IEEE Pers. Commun. Mag.: Special Issue on Advances in Mobile Ad Hoc Networking, vol. 8, Jan. 2001.

[15] P. Johansson et al., "Scenario-based performance analysis of routing protocols for mobile ad hoc networks," in Proc. IEEE/ACM MOBICOM, 1999, pp. 195-206.

[16] D. Johnson and D. Maltz, "Dynamic source routing in ad hoc wireless networks," in Mobile Computing, T. Imielinski and H. Korth, Eds. Norwell, MA: Kluwer, 1996, ch. 5, pp. 153-181.

[17] R. Kahn, "The organization of computer resources into a packet radio network," IEEE Trans. Commun., vol. COM-25, pp. 169-178, Jan. 1977.

[18] R. Kahn et al., "Advances in packet radio technology," Proc. IEEE, vol. 66, pp. 1468-1496, Nov. 1978.

[19] J. Laneman and G. Wornell, "Energy-efficient antenna sharing and relaying for wireless networks," in Proc. IEEE Wireless Communications, Networking Conf., 2000, pp. 7-12.

[20] - "Exploiting distributed spatial diversity in wireless networks," in Proc. Allerton Conf. Communications, Control, Computing, 2000, pp. $1-10$.

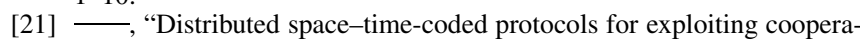
tive diversity in wireless networks," IEEE Trans. Inform. Theory, vol. 49, pp. 2415-2425, Oct. 2003.

[22] J. Laneman, D. Tse, and G. Wornell, "Cooperative diversity in wireless networks: Efficient protocols and outage behavior," IEEE Trans. Information Theory, submitted for publication.

[23] J. Proakis, Digital Communications, 4th ed. New York: McGraw-Hill, 2001.

[24] C. Qiao, H. Wu, and O. Tonguz, "Load balancing via relay in next-generation wireless systems," in Proc. Mobile, Ad Hoc Networking, Com puting Conf., 2000, pp. 149-150.

[25] T. Rappaport, Wireless Communications: Principles \& Practice. Englewood Cliffs, NJ: Prentice-Hall, 1996.

[26] M. Schwartz, W. R. Bennett, and S. Stein, Communication Systems and Techniques. New York: McGraw-Hill, 1966.

[27] A. Sendonaris, E. Erkip, and B. Aazhang, "User cooperation diversity-Part I: System description," IEEE Trans. Commun., vol. 51, pp. 1927-1938, Nov. 2003.

[28] - "User cooperation diversity—Part II: Implementation aspects and performance analysis," IEEE Trans. Commun., vol. 51, pp. 1939-1948, Nov. 2003

[29] V. Sreng, H. Yanikomeroglu, and D. Falconer, "Coverage enhancement through two-hop relaying in cellular radio systems," in Proc. IEEE Wireless Communications, Networking Conf., vol. 2, 2002, pp. 881-885.

[30] V. Wong and C. Leung, "A transmit power control scheme for improving performance in a mobile packet radio system," IEEE Trans. Veh. Technol., vol. 43, pp. 174-180, Jan. 1994.

[31] L. Yang, H. Hasna, and M.-S. Alouini, "Average outage duration of multihop communication systems with regenerative relays," in Proc. IEEE Vehicular Technology Conf., vol. 1, 2003, pp. 171-175.

[32] J. Zander, "Radio resource management in future wireless networks: Requirements and limitations," IEEE Commun. Mag., pp. 30-36, Aug. 1997.

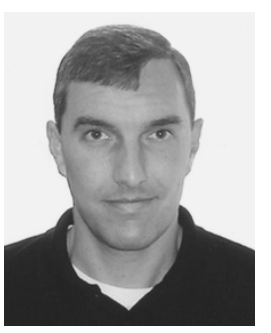

John Boyer (S'99) was born in Ottawa, ON, Canada, on March 30, 1976. He received the B.Eng. degree in computer system engineering and the M.A.Sc. degree in electrical engineering in 1999 and 2001, respectively, from Carleton University, Ottawa, ON, Canada, where he is currently working toward the $\mathrm{Ph} . \mathrm{D}$. degree in electrical engineering.

His research interests are in wireless communications systems, with a particular focus on multihop, mesh, and relaying networks. Since 2001, he has been with ActivCard Corporation, Fremont, CA, a leading global provider of digital identity and authentication solutions, where he holds the position of Senior Manager, Architecture. In this capacity, he leads an international team with responsibility for driving the design of ActivCard's products and solutions. He holds a number of patents granted and pending in the fields of enterprise security and authentication. 


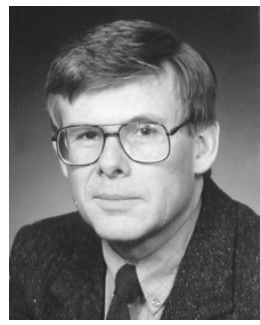

David D. Falconer (M'68-SM'83-F'86) received the B.A. Sc. degree in engineering physics from the University of Toronto, Toronto, ON, Canada, and the S.M. and Ph.D. degrees in electrical engineering from the Massachusetts Institute of Technology, Cambridge.

After a year as a Postdoctoral Fellow at the Royal Institute of Technology, Stockholm, Sweden, he was with Bell Laboratories, Holmdel, NJ, from 1967 to 1980, as a Member of the Technical Staff and later as Group Supervisor. During 1976-77, he was a Visiting Professor at Linköping University, Linköping, Sweden. Since 1980, he has been with Carleton University, Ottawa, ON, Canada, where he is Chancellor's Professor in the Department of Systems and Computer Engineering. His current research interests center around beyond-third-generation broadband wireless communications systems. He is currently Director of the Broadband Communications and Wireless Systems Centre (BCWS) at Carleton University. He led a CITR broadband wireless research project, involving a number of Canadian universities and companies from 1990 to 1998, and was an active contributor to the IEEE 802.16 wireless MAN standards group in 1999-2001. $\mathrm{He}$ is currently Chair of Working Group 4 (New Radio Interfaces, Relay-Based Systems and Smart Antennas) of the Wireless World Research Forum (WWRF).

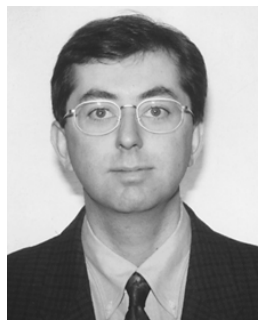

Halim Yanikomeroglu (S'97-M'99) was born in Giresun, Turkey, in 1968. He received the B.Sc. degree in electrical and electronics engineering from the Middle East Technical University, Ankara, Turkey, in 1990, and the M.A.Sc. degree in electrical engineering (now ECE) and the Ph.D. degree in electrical and computer engineering from the University of Toronto, Toronto, ON, Canada, in 1992 and 1998, respectively.

He was with the Research and Development Group of Marconi Kominikasyon A.S., Ankara, Turkey, from January 1993 to July 1994. Since 1998, he has been with the Department of Systems and Computer Engineering at Carleton University, Ottawa, ON, Canada, where he is now a tenured Associate Professor. His research interests include almost all aspects of wireless communications with a special emphasis on infrastructure-based multihop/mesh/relay networks, radio resource management, and multiantenna systems.

Dr. Yanikomeroglu has been involved in the steering committees and technical program committees of numerous international conferences in wireless communications; he has also given several tutorials in such conferences. He was the Technical Program Co-Chair of the IEEE Wireless Communications and Networking Conference 2004 (WCNC'04). He is an editor for IEEE TRANSACTIONS ON WiRELESS COMMUNICATIONS, and a Guest Editor for the Wiley Journal on Wireless Communications \& Mobile Computing; he was an Editor for IEEE Communications Surveys and Tutorials for 2002-2003. Currently, he is serving as the Vice-Chair of the IEEE Technical Committee on Personal Communications. He is a registered Professional Engineer in the Province of Ontario, Canada. 